\title{
Arzneimittel aus dem Internet
}

\author{
Von Schweizer Patienten werden immer mehr Medikamente im Internet be- \\ stellt. Das bringt Gesundheitsgefahren mit sich. Auch gefälschte, wirkungs- \\ lose oder gesundheitsschädigende Produkte werden vertrieben.
}

Ruth Mosimann

Korrespondenz:

Ruth Mosimann

Swissmedic,

Schweizerisches Heilmittelinstitut

Einheit Kontrolle illegale

Arzneimittel

Hallerstrasse 7

Postfach

CH-3000 Bern 9

Tel. 0313220472

Fax 0313220212

ruth.mosimann@swissmedic.ch

www.swissmedic.ch

\section{Importe von Medikamenten / Massnahmen der Behörden}

Am Schweizer Zoll werden illegale Importe blockiert. Obwohl die wenigsten Sendungen als Medikamentensendungen deklariert sind, werden durch Mitarbeiter am Zoll regelmässig illegale Arzneimittelimporte entdeckt. Nach der Beschlagnahmung der Ware am Zoll eröffnet Swissmedic ein Verwaltungsverfahren, das den Verlust (meist die Vernichtung) der Arzneimittel zur Folge hat. Die Kosten dieses Verfahrens liegen bei mindestens 300 Franken und müssen vom Besteller in der Schweiz getragen werden.

Gemäss Heilmittelgesetz ist es möglich, Arzneimittel in kleinen Mengen (innerhalb eines Monatsbedarfs) für den Eigengebrauch legal zu importieren. Diese ursprünglich für einreisende Touristen gedachte Bestimmung wird vermehrt auch von Schweizern benutzt, um sich rezeptpflichtige Arzneimittel via Internet aus dem Ausland zu besorgen. In einer kürzlich durchgeführten Aktion wurde festgestellt, dass $27 \%$ aller von Indien und $16 \%$ aller von Thailand importierten Postpäckchen Arzneimittel enthielten. 40\% dieser Importe durch Privatpersonen lagen innerhalb der legalen Menge.

\section{Qualität}

Die Qualität der im Internet bestellten Arzneimittel ist sehr unterschiedlich. Es besteht die Gefahr, dass gefälschte Medikamente geliefert werden. Arzneimittelfälschungen sind Medikamente, die bezüglich der Zusammensetzung oder auch der Herkunft absichtlich falsche Angaben tragen. Oft enthalten gefälschte Arzneimittel keinen Wirkstoff, einen falschen Wirkstoff oder den Wirkstoff in Unter- oder Überdosierung. Sie wurden unter nichtkontrollierten Bedingungen hergestellt und klinisch nicht geprüft.

Bei einzelnen Medikamentengruppen werden auch Imitationen importiert. Diese illegalen Imitationen sind Medikamente, die von der Aufmachung her nicht versuchen, ein Originalprodukt direkt zu kopieren. Sie enthalten aber oft den gleichen Wirkstoff oder präsentieren sich bezüglich Aussehen ähnlich wie das Original.

\section{Unerwünschte Wirkungen von illegalen Arzneimitteln}

Dieses Jahr waren in Asien mehrere Erektionsförderer im Markt, die anstelle von potenzfördernden Wirkstoffen das orale Antidiabetikum Glibenclamid in überhöhter Dosierung enthielten. Die Behörde von Singapur wurde dank der Meldung einer unerwünschten Wirkung auf diese Fälschungen aufmerksam: Ein Patient war mit einer schweren Hypoglykämie in ein Spital eingeliefert worden und verstorben. Auch in der Schweiz wurde vor wenigen Wochen eine gesundheitsgefährdende Fälschung entdeckt: «Powertabs» wurde als rein pflanzliches Potenzmittel beworben. Swissmedic stellte in Analysen jedoch fest, dass in «Powertabs» ein dem Sildenafil verwandter, nirgends zugelassener Wirkstoff enthalten war.

Angesichts der relativ grossen Menge an Arzneimitteln, die von Privatpersonen via Internet bezogen werden, appelliert Swissmedic an die Ärzteschaft, bei unerwünschten Wirkungen oder unerwarteten Zwischenfällen bei Patienten auch importierte Arzneimittel in Betracht zu ziehen. Bitte benutzen Sie das gelbe Formular zur Mel-

\section{Abbildung 1}

Illegal importierte Erektionsförderer: Fälschungen (links) und Imitationen (rechts).

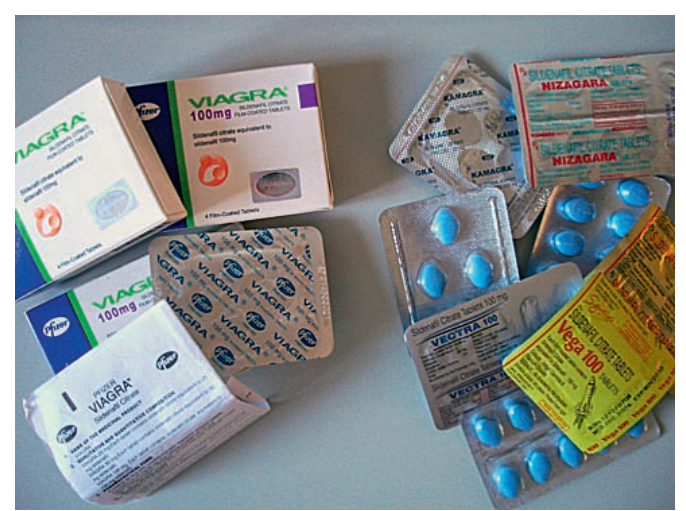


dung unerwünschter Arzneimittelwirkungen, auch wenn nichtzugelassene oder illegal importierte Arzneimittel die Ursache einer UAW sind. Das Meldeformular ist verfügbar auf der Homepage von Swissmedic (www.swissmedic.ch unter Marktüberwachung $\rightarrow$ Pharmacovigilance $\rightarrow$ Gesamtüberblick $\rightarrow$ Meldeformular), im Anhang des Arzneimittelkompendiums oder kann bei Swissmedic über Tel. 0313220223 bestellt werden.

\section{Publikumskampagne}

Obwohl Swissmedic illegale Importe bekämpft und immer öfter auch mit ausländischen Behörden gegen illegale Internetapotheken zusammenarbeitet, kann die Medikamentenflut aus dubiosen Quellen nicht ursächlich gestoppt werden. Sehr wichtig ist es, bei der Schweizer Bevölkerung das Bewusstsein dafür zu verstärken, dass Medikamente nicht im Internet bestellt werden sollten.

Swissmedic warnt regelmässig in Pressemitteilungen vor gefährlichen Medikamenten. Dieses Jahr wurde ein aktualisierter Leitfaden auf der Website von Swissmedic aufgeschaltet, der über Risiken und gesetzliche Grundlagen betreffend die Bestellung von Arzneimitteln im Internet informiert. Ausserdem initiierte Swissmedic zusammen mit Interpharma eine Publikumskampagne unter dem Dach von STOP PIRACY. Die Kampagne läuft über verschiedene Medien und gipfelt im STOP PIRACY DAY am 25. Oktober 2008, an dem Kunden u.a. in mehr als 500 Schweizer Apotheken ihre im Internet bestellten Arzneimittel auf Echtheit überprüfen lassen können.

\section{Abbildung 2}

Logo des Aktionstages mit Fälschungscheck in Apotheken.

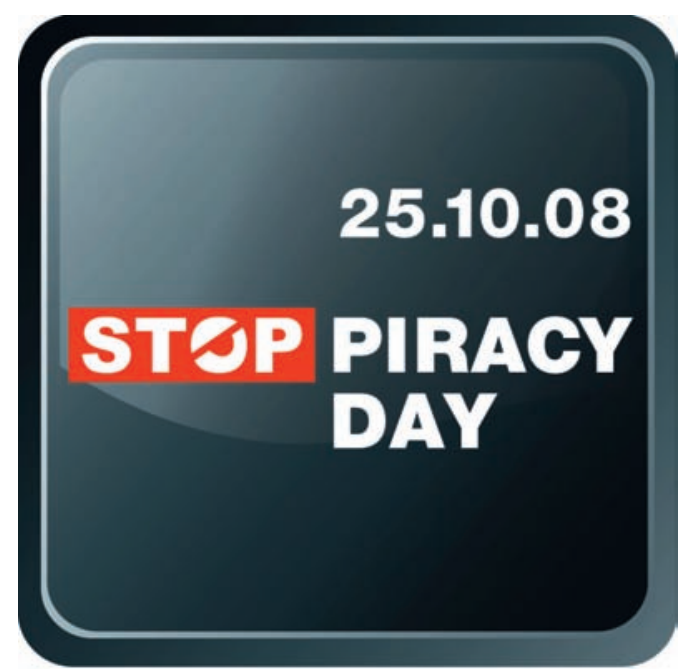

\section{Importe von Medikamenten}

Swissmedic schätzt, dass pro Jahr mindestens 50000 Sendungen von Privatpersonen illegal in die Schweiz importiert werden.

In Zusammenarbeit mit dem Zoll beschlagnahmt Swissmedic illegale Sendungen. Darin befinden sich oft:

- Erektionsförderer;

- Muskelaufbaupräparate;

- Medikamente zum Gewichtsverlust;

- andere rezeptpflichtige Präparate. 